\title{
Abordaje de lesiones quísticas de páncreas
}

\author{
Ana C. Lira-Treviño ${ }^{1}$ y Mario Peláez-Luna ${ }^{1,2 *}$ \\ ${ }^{1}$ Departamento de Gastroenterología, Instituto Nacional de Ciencias Médicas y Nutrición Salvador Zubirán; ²División de Investigación, Facultad \\ de Medicina, Universidad Nacional Autónoma de México. Ciudad de México, México
}

\begin{abstract}
Resumen
El manejo conservador o quirúrgico de las NQP es establecido por el diagnóstico definitivo de estas. Además de la historia clínica y características de imagen, el análisis del líquido del quíste, biopsia de sus paredes y tejido circundante ayudan a establecer la naturaleza benigna (ej. pseudoquiste, cistadenoma seroso), maligna (p. ej., degeneración quística de adenocarcinoma de páncreas) y potencialmente malignos (p. ej., neoplasias mucinosas papilares intraductales; IPMN). Las IPMN son las NQP más frecuentes; sus características clínicas y del quiste establecen el riesgo de progresión maligna. A continuación se sintetizan los trabajos más relevantes sobre el abordaje diagnóstico de neoplasias quisticas de páncreas (NQP) presentados en la Semana de Enfermedades Digestivas (DDW) 2020 de la Asociación Americana de Gastroenterología.
\end{abstract}

Palabras clave: Neoplasia quistica de páncreas. Ultrasonido endoscópico. Biopsia por aspiración. Endomicroscopia confocal. Antígeno carcinoembrionario.

Mediante el acceso al sitio de internet: https://ddw. apprisor.org/epsSearchDDW.cfm realizamos una búsqueda de los resúmenes presentados en la DDW 2020 sobre abordaje de NQP y seleccionamos aquellos que consideramos los más relevantes.

Se desarrolló una herramienta de estratificación de riesgo denominada DART-1 para identificar IPMN de bajo riesgo. Esta considera diferentes factores de riesgo ponderados y proporciona un puntaje que predice la posibilidad de desarrollar datos preocupantes 0 alto riesgo 3 a 5 años posterior al diagnóstico de IPMN. En una cohorte de pacientes con IPMN sometidos a vigilancia durante 3 años, un puntaje de DARTT-1 menor/igual a 4.94 sugiere una baja probabilidad de progresión ${ }^{1}$.

Un aspecto relevante del abordaje de las NQP es determinar si producen o no mucina. Esto se logra con la determinación de antígeno carcinoembrionario (ACE) en el líquido del quiste. Es importante recordar que el ACE no diferencia entre cistadenoma mucinoso (CAM) e IPMN y tampoco detecta transformación maligna. Aún y cuándo un estudio retrospectivo encontró diferencias en los niveles de ACE entre los CAM e IPMN de alto riesgo y los IPMN de bajo riesgo, los casos son altamente seleccionados y escasos ${ }^{2}$. La determinación de glucosa distingue neoplasias mucinosas y no mucinosas; las primeras presentan niveles menores. Puntos de corte de $30 \mathrm{mg} / \mathrm{dl} \mathrm{y}<50 \mathrm{mg} / \mathrm{dl}$ tuvieron una sensibilidad de $89.4 \%$, especificidad de $76.2 \%$ para distinguir entre estas lesiones ${ }^{3,4}$.

La incapacidad del ACE y glucosa para identificar lesiones malignas y premalignas pudiera resolverse mediante estudios genéticos. Estos han reportado diferencias en la frecuencia de mutaciones en genes KRAS/GNAS/RNF43, que ayudan a diferenciar IPMN 
de CAM con $93 \%$ de sensibilidad y $100 \%$ de especificidad. La presencia de mutaciones en genes TP53/ SMAD4/mTOR identifican aquellos IPMN con neoplasia avanzada con una sensibilidad de $96 \%$ y especificidad de $100 \%$. Otras mutaciones ayudan a identificar tumores neuroendocrinos con degeneración quística (MEN1/CNA) y cistadenoma seroso $(\mathrm{VHL})^{5,6}$.

Además de su utilidad en el diagnóstico diferencial, el análisis del material genético puede identificar transformación (pre) maligna y sugerir la periodicidad de vigilancia de las NQP. Un estudio realizó análisis serial de $A D N$ en el líquido del quiste a 3332 personas con NQP. De acuerdo a la cantidad de anormalidades en el ADN (A-ADN), los pacientes fueron categorizados como aquellos con 0, 1 (no preocupante), o múltiples (2-3; preocupante) A-ADN que incluían: cantidad elevada de ADN, mutaciones KRAS y perdida de heterocigosidad del gen supresor de tumores (TSG-LOH). Al momento del diagnóstico, 69.5\% no presentaba A-ADN, 27.7\% tuvo 1 A-ADN y $2.8 \%>2$ A-ADN. De aquellos sin A-ADN inicial, $81 \%$ permaneció estable, $18 \%$ desarrolló 1 A-ADN y $1 \%>2$ A-ADN durante un seguimiento de 1.4-1.6 años. Del grupo con 1 A-ADN inicial, $61 \%$ se mantuvo estable; el $35 \%$ y $4 \%$ desarrollaron 1 y $>2$ A-ADN respectivamente en un seguimiento de 1.1 a 1.5 años. El incremento de A-ADN fue mayor en el grupo con > 2 A-ADN inicial; solo $38 \%$ permaneció estable mientras que 41 y $20 \%$ desarrollaron 1 y $>2$ A-ADN en un seguimiento de 6 a 18 meses. En los grupos con 1 y $>2$ A-ADN iniciales, $61 \%$ y $38 \%$ presentaron regresión a 0 ADN respectivamente. Estos hallazgos sugieren que aquellos pacientes sin A-ADN preocupantes (0-1) continuarán estables los siguientes 18 meses al diagnóstico y su vigilancia puede extenderse a $>1$ año. Aun y cuando las A-ADN puede desaparecer, la mayoría de los casos con > 2 A-ADN persiste y mantiene sus características de alto riesgo ${ }^{7}$.

Considerando las limitaciones diagnósticas y económicas del análisis químico y genético, se han desarrollado instrumentos que permitan obtener mayor cantidad de tejido. Un metaanálisis y un estudio retrospectivo reportaron un mayor rendimiento diagnóstico de la biopsia por microfórceps (BMF) guiada por USE comparada con la biopsia por aspiración con aguja fina (BAAF) 82 vs. $59.1 \%$, respectivamente y una razón de momios de 3.88 (IC 95\%: 1.75-8.53) en favor de BMF. La BMF también fue superior a ACE y mutaciones y no se asoció a mayores complicaciones ${ }^{8,9}$.

La endomicroscopia confocal (EC) desarrollada para evaluar la mucosa del tracto gastrointestinal, se ha adaptado al estudio de el epitelio de las NQP. Krishna, et al. desarrollaron una herramienta computacional mediante aprendizaje profundo (deep-lerning) que identifica IPMN con displasia grado bajo/intermedio (DBG) y de alto grado/Cáncer (DAG/Ca). Utilizaron videos de EC en IPMN tratados quirúrgicamente (19 DAG/Ca y 17 DBG) y diseñaron 2 algoritmos basados en redes neurales convolucionadas. Un modelo holístico que considera todas las características de las IPMN observadas con EC y un modelo de segmentación en el que el sistema de detección computacional es entrenado para graduar la displasia en base al grosor y oscuridad de las papilas epiteliales. El sistema holístico diferenció DAG/Ca y DBG con una precisión de $86 \%$, sensibilidad de $83 \%$ y especificidad de $88 \%$. El modelo de segmentación tuvo una precisión de $83 \%$, sensibilidad de $83 \%$ y especificidad de $82 \%$. Los mismos autores realizaron un análisis costo-beneficio de la EC en la evaluación de NQP $>2 \mathrm{~cm}$. Encontraron desacuerdo diagnóstico entre el manejo estándar y la EC en $14 \%$ de los casos. Agregar la EC representó ahorros totales por USD $\$ 381,337.00$ (USD $\$ 4,100.00$ por paciente) asociado a una mejor identificación de neoplasias no mucinosas ${ }^{10,11}$.

\section{Conclusiones}

1. El modelo de predicción DART-1 para IPMN detecta satisfactoriamente lesiones con bajo riesgo a desarrollar características preocupantes o de alto riesgo.

2. Los niveles de ACE y glucosa en líquido de NQP diferencia lesiones mucinosas de no mucinosas.

3. Mutaciones genéticas de genes específicos en el líquido de NQP tiene un rendimiento diagnóstico adecuado.

4. La BMF y la EC son herramientas potencialmente costo-efectivas que pueden mejorar el abordaje y diagnóstico diferencial de las NQP.

\section{Financiamiento}

Los autores no recibieron patrocinio para llevar a cabo este artículo.

\section{Conflicto de intereses}

Los autores declaran no tener conflicto de intereses alguno.

\section{Bibliografía}

1. Phan J, Thiruvengadam S, Kim S., et al. Application of a risk stratification tool to identify low risk intraductal papillary mucinous neoplasms on a prospective cohort. Presentada en: DDW; 2020 Mayo 2-5; Chicago IL. Su1488. 
2. Köker I, Ünver N, Malya F, et al. Cyst fluid carcinoembryionic antigen difference among mucinous neoplasms and intraductal papillary mucinous neoplasms. Presentada en: DDW; 2020 Mayo 2-5; Chicago IL. Sa1443.

3. Singhi A, Nikiforova M, McGrath $\mathrm{K}$, et al. Next-generation sequencing of pancreatic cyst fluid is both sensitive and specific for the classification of neoplastic cysts and the detection of advanced neoplasia in intraductal papillary mucinous neoplasms: a prospective multi-institutional study Presentada en: DDW; 2020 Mayo 2-5; Chicago IL. Sa1396.

4. McCarty T, Paleti S, Rustagi T. Molecular analysis of EUS- acquired pancreatic cyst fluid for KRAS and GNAS mutations for diagnosis of intraductal papillary mucinous neoplasia and mucinous cystic lesions: A systematic review and meta-analysis. Presentada en: DDW; 2020 Mayo 2-5; Chicago IL. Su1500.

5. Rossi G, Petrone M, Cupurso G, et al. Glucose levels in EUS- Aspirated Cyst fluid have a high accuracy for the diagnosis of mucinous pancreatic cystic lesions. Presentada en: DDW; 2020 Mayo 2-5; Chicago IL. Sa1421.

6. Oria I, Lariño-Noia J, Villaverde A, et al. Cyst fluid glucose obtained by EUS-FNA is accurate for the diagnosis of mucinous pancreatic cysts. Experience from two tertiary care centers. Presentada en: DDW; 2020 Mayo 2-5; Chicago IL. Sa1413.
7. Chandrasekhara V, Jackson S, Toney N, et al. Molecular stability, regression and progression in pancreatic cysts over time. Presentada en: DDW; 2020 Mayo 2-5; Chicago IL. Su 1487.

8. Nutalapati V, Chandrasekar T, Dasari C, et al. EUS-Guided through the needle micro-forceps biopsy versus EUS-Guided Fine needle aspiration in the evaluation of pancreatic cysts: A systematic review and meta-analysis. Presentada en: DDW; 2020 Mayo 2-5; Chicago IL. Sa1408.

9. Chakta D, De Latour R, Harber G, et al. Endoscopic ultrasound-guided through-the-needle biopsy of pancreatic cystic lesions: diagnostic outcomes and adverse events. Presentada en: DDW; 2020 Mayo 2-5; Chicago IL. Sa1479.

10. Krishna S, Hart P, Papachristou G, Porter K, et al. The impact of endoscopic ultrasound-guided confocal endomicroscopy for pancreatic cystic lesions on healthcare costs. Presentada en: DDW;2020 Mayo 2-5; Chicago IL. Su 1493.

11. Krishna S, Chao W, Poland S, et al. Computer-aided detection of advanced neoplasia in intraductal papillary mucinous neoplasms using confocal laser endomicroscopy. Presentada en: DDW; 2020 Mayo 2-5; Chicago IL. 266. 